\title{
Berichtigung zu A. N. 2835 und 3086.
}

In A. N. 2835 und 3086 sind die Formeln mitgetheilt worden, welche zur Reduction der mit einen Lamellenmikrometer angestellten Beobachtungen dienen können. In den für die Berechnung der Refractionscorrection geltenden Ausdricken ist nun ein Versehen vorgekommen, auf welches mich Herr Dr. Ristenpart gefälligst aufmerksan gemacht hat, indem nämlich die Differentialquotienten nach $t$ mit umgekehrtem Vorzeichen in die Gleichungen eingefübrt worden sind. Dieser Irrthum ist dadurch entstanden, dass ursprünglich die andere Lage als Lage \& bezeichnet wurae, so dass $\mathrm{d} t=\mp \mathrm{d} d \sec \delta$ zu setzen war. Später wurde die Lagenbezeichnung aus formalen Gründen geändert, es mussite dann also, wie auch a. a. $O$. angegeben ist, $\mathrm{d} t= \pm \mathrm{d} d$ sea $\delta$ gesetzt werden; aus Versehen ist aber die frthere Gleichung benutzt worden. Wird dieser Fehler verbessert, so ergiebt sich unter Beibehaltung der für die Refractionscorrection eingefuhrten Form

$$
\begin{aligned}
& \Delta(\mathrm{d} \alpha)=1 / 2\left(P_{1}+P_{2}\right)+1 / 2\left(Q_{1}-Q_{2}\right) \sec \delta_{0} \\
& \Delta(\mathrm{d} \delta)=1 / 2\left(Q_{1}+Q_{3}\right)+1 / 2\left(P_{1}-P_{2}\right) \cos \delta_{0}
\end{aligned}
$$

in Uebereinstimmung mit den von Herrn Dr. Ristenpart (A. N. 3194) erhaltenen Ausdrücken :

$$
\begin{aligned}
& P=-\frac{2 k \mathrm{~d} d \operatorname{ctg} n \sin \delta_{0}}{\cos ^{2} \delta_{0} \sin \left(N+\delta_{0}\right)} \\
& Q=\frac{k \mathrm{~d} d}{\sin ^{2}\left(N+\delta_{0}\right)}\left(\cos N \cos \left(N+\delta_{0}\right) \sec \delta_{0}-\operatorname{ctg}^{2} n\right) .
\end{aligned}
$$

Potsdam 1893 Dec. 4 .

P. Kempf.

\section{Notiz betr. Nova (T) Aurigae.}

Eben mache ich die interessante Entdeckung, dass sich auf einer Nordlichtphotographie, weiche ich 1892 Jan. 5 an der nördlichen Küste Norwegens erhalten habe, die Nova Aurigae befindet. Ich hatte mir schon seit Jahr und Tag vorgenommen, meine Platten daraufhin durchzusehen, bro aber anderer Sachen wegen erst jetzt zur Bearbeitung derselben gekommen.

Die Nova befindet sich leider nahe am Rande der Platte, $\chi$ Aurigae ist nicht mehr darauf; sie ist aber ziemlich deutlich zu sehen, da zum Theil Sterne unter der 8. Grösse noch gerade zu erkeanen sind. Die Aufaahme ist mit einer orthochromatischen gelbgrünempfindlichen Platte gemacht.

Greifswald 1893 Dec. 9.

\section{Martin Brendel.}

\section{Numerirung und Benennung von kleinen Planeten.}

Ausser der in A. N. 3194 mitgetheilten Liste sind im Berl. Astr. Jahrb. für 1896 noch die folgenden neuen Planeten numerirt worden:

\begin{tabular}{cccc|crcc} 
Planet & entdeckt & Entdecker & Nr. & Planet & entdeckt & Entdecker & Nr. \\
1893 AJ & Sept. 15 & Charlois & $(373)$ & 1893 AM & Sept. 18 & Charlois & $(376)$ \\
AK & -18 & & $(374)$ & AN & 20 & & $(377)$ \\
AL & $>18$ & & $(375)$ & & & &
\end{tabular}

Ferner hat der Planet (344), entdeckt 1892 Nov. 15 von Charlois in Nizza, den Namen Desiderata erhalten، Kr.

\section{Entdeckung von zwei neuen Planeten 1894 AT und AD.}

Deux planetes Charlois 29 Janvier:

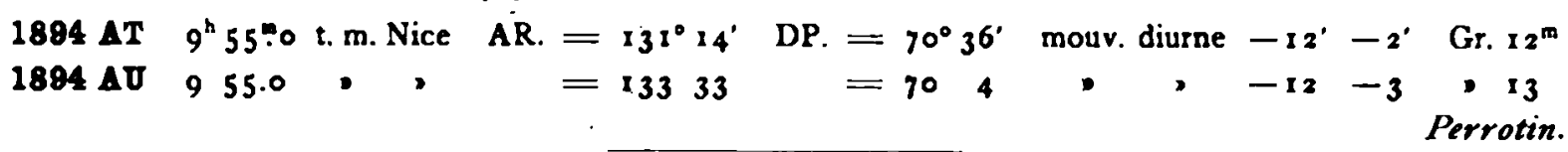

Berichtigung zu Nr. 3208 Bd. 134 p. 258 Tabelle unten, letzte Col.: +151 ist eine Zeile tiefer zu rücken.

Inhalt zu Nr. 3209. S. Kostinsky. Nouvelles recherches sur les variations de la, latitude de Poulkovo. 265. - E. Becker. Vergleichang der in Strassburg beobachteten Breitenschwanlungen mit der Rechnung. 271 . - F. Porro. Sulla declinazione di 33 Bootis. 273. - A. Rició. Sulla percezione più rapida delle stelle più luminose. 277. - P. Kempf. Berichtigung zil A. N. 2835 und 3086. 279. - M. Brendel. Notiz betr. Nova (T) Aurigae. 279. - Numerirung und Benennung von kleinen Planeten. 279. Perrotin. Entdeckung von 2 wei neuen Planeten 1894 AT und AU. 279. - Berichtigung. 279. 